SU-4240-587

UNITU-THEP-22/1994

October 1994

hep-ph/9410321

\title{
Resolving Ordering Ambiguities in the Collective Quantization by Particle Conjugation Constraints
}

\author{
J. Schechter ${ }^{a}$ and H. Weigel ${ }^{a, b}$ \\ ${ }^{a}$ Department of Physics, Syracuse University \\ Syracuse, NY 13244-1130, USA \\ ${ }^{b}$ Institute for Theoretical Physics, Tübingen University \\ D-72076 Tübingen, Germany
}

\begin{abstract}
We formulate the particle conjugation operation and its convenient realization as $G^{-}$ parity in the framework of several chiral soliton models. The Skyrme model, the Skyrme model with vector mesons and the chiral quark model are specifically treated. The vector and axial vector currents are classified according to their behavior under $G$-parity. In the soliton sector particle conjugation constrains a priori ambiguous orderings of operators in the space of the collective coordinates. In the Skyrme model with vector mesons and in a local chiral model with an explicit valence quark this classification scheme provides consistency conditions for the ordering of the collective operators appearing in the $1 / N_{C}$ corrections to the nucleon axial charge and the isovector magnetic moment. These consistency conditions cause the corrections obtained from an ordinary perturbation expansion to vanish in the context of the collective quantization of the static soliton configuration. This conclusion presumably
\end{abstract}


applies to all local effective chiral models. 


\section{Introduction}

In this paper we shall discuss some theoretical constraints on the vector and axial vector current matrix elements of the nucleon predicted in various kinds of chiral soliton models (for review articles see [1], 2]). One might, at first, think that this is a subject which has been completely exhausted. However, there are, at least, two recent problems:

i) the "too small $g_{A}$ problem" [1]

ii) the "proton spin puzzle" [3]

where such considerations appear crucial. These problems involve current matrix elements which are of subleading order in an appropriate perturbation approach to the collective Hamiltonian describing the nucleon. As such, they turn out to involve operator ordering ambiguities. Symmetries like iso-spin invariance do not seem to be able to offer guidance on this question. Here we point out that particle conjugation symmetry can, as it does in ordinary field theory, provide operator ordering restrictions. For example, charge conjugation invariance requires the QED interaction to have the ordered form $\frac{1}{2} e A^{\mu}\left(\bar{\Psi} \gamma_{\mu} \Psi-\Psi^{T} \gamma_{\mu}^{T} \bar{\Psi}^{T}\right)$.

Recently, the very interesting observation has been made [4]- [9] that the too small value of the neutron beta decay constant $g_{A}$ predicted in many chiral soliton models might be dramatically improved by including $1 / N_{C}$ corrections. This seems satisfying both because it agrees with the quark model result [10] in which $g_{A}$ should behave like $\left(N_{C}+2\right) / 3$ so sizable corrections are expected and because the corrections fit the pattern expected from unitarity constraints on pion-nucleon matrix elements [11] (which imply that the structure of the leading and next-to leading order terms is identical). In spite of these pleasing features, we will see that the imposition of proper ordering constraints requires that these $1 / N_{C}$ corrections vanish in certain chiral soliton models.

Actually, a different problem with these corrections has already been noted [12]. The equation of motion for the pion fields, which yields the PCAC (Partially Conserved Axial Current) relation, is derived from the static energy functional of the soliton. This functional is of the order $N_{C}$ without any subleading corrections. Thus this equation of motion cannot account for $1 / N_{C}$ corrections in the divergence of the axial current. The pion field develops a Yukawa-type tail whose amplitude is, due to PCAC, proportional to $g_{A}$. This allows one to not only compute $g_{A}$ by direct integration of the axial current but also from the large distance behavior of the pion [13. The latter, however, is completely determined from the classical equation of motion and hence does not contain the $1 / N_{C}$ corrections. Therefore it has been concluded that these corrections violate PCAC by about 30\% [12]. This problem was circumvented by elevating the derivative of the axial current including the corrections to the equation of motion via PCAC [12, 6]. However, this treatment is not completely satisfactory since it has not proven possible to derive the so constructed equation of motion from an action principle.

We shall, for simplicity, work in a theory with just the two lightest quark flavors present. The relevant currents at the quark level are the vector and axial vector ones of both isoscalar and and isovector type

$$
\begin{aligned}
V_{\mu}^{0} & =\frac{1}{2}\left(\bar{q} \gamma_{\mu} q-q^{T} \gamma_{\mu}^{T} \bar{q}^{T}\right), \quad V_{\mu}^{a}=\frac{1}{4}\left(\bar{q} \gamma_{\mu} \tau^{a} q-q^{T} \gamma_{\mu}^{T} \tau^{a} \bar{q}^{T}\right) \\
A_{\mu}^{0} & =\frac{1}{2}\left(\bar{q} \gamma_{\mu} \gamma_{5} q-q^{T} \gamma_{\mu}^{T} \gamma_{5}^{T} \bar{q}^{T}\right), \quad A_{\mu}^{a}=\frac{1}{4}\left(\bar{q} \gamma_{\mu} \gamma_{5} \tau^{a} q-q^{T} \gamma_{\mu}^{T} \gamma_{5}^{T} \tau^{a} \bar{q}^{T}\right)
\end{aligned}
$$

where $q$ represents the quark field. Notice that all of these operators are hermitean. In dealing with the charge conjugation of objects with definite isospin transformation properties, 
it is often useful to define the concept of $G$-parity [16]. Technically the $G$-parity reflection is defined as charge conjugation followed by a rotation of angle $\pi$ around the $y$-axis in isospace. This corresponds to a unitary operator $\mathcal{G}=\mathrm{e}^{i \pi I_{2}} \mathcal{C}$. Since the $G$-parity of the pion is minus one, the $G$-parity classification provides a tool to determine whether the number of pions emerging from a decay process is even $(G$-parity $=1)$ or odd $(G$-parity $=-1)$. By construction the $G$-parity is a conserved quantity for interactions which are invariant under charge conjugation and isospin rotations. For the current operators in (1.1) we have

$$
\mathcal{G}\left(V_{\mu}^{0}, V_{\mu}^{a}, A_{\mu}^{0}, A_{\mu}^{a}\right) \mathcal{G}^{-1}=\left(-V_{\mu}^{0}, V_{\mu}^{a}, A_{\mu}^{0},-A_{\mu}^{a}\right)
$$

In chiral models the pions are incorporated via the non-linear realization $U=\exp (i \boldsymbol{\pi}$. $\left.\boldsymbol{\tau} / f_{\pi}\right)$ where $f_{\pi}=93 \mathrm{MeV}$ denotes the weak pion decay constant. Since $\boldsymbol{\pi} \rightarrow-\boldsymbol{\pi}$ under the $G$-transformation we simply have

$$
U(\boldsymbol{r}, t) \stackrel{G}{\longrightarrow} U^{\dagger}(\boldsymbol{r}, t) .
$$

One should bear in mind that in all quark soliton models the quark fields are functionals of the chiral field so their behavior under conjugation is determined from that of $U$.

This paper is organized as follows. In section 2 the behavior of the symmetry currents in the simple Skyrme model under $G$-parity reflection will be studied. This will be generalized to models with vector mesons in section 3 . In section 4 we will first discuss the particle conjugation in the framework of quark soliton models and then apply these results to the $1 / N_{C}$ corrections to $g_{A}$ and $\mu_{V}$.

Actually the problem of ordering ambiguities in soliton models was already discussed by Tomboulis [14 in the case of a two dimensional field theory. Also in that reference symmetry arguments (Poincaré invariance) have been employed to resolve operator ordering ambiguities. However, we will mainly be concerned with form factors at zero momentum transfer and hence do not introduce collective coordinates for the boost of the soliton. Rather we are interested in ordering ambiguities for the collective coordinates associated with internal symmetries ${ }^{a}$.

\section{G-Parity Constraints on the Currents in the Skyrme Model}

In order to introduce the relevant background and notation we start with the simple Skyrme model, defined by the Lagrangian 17, 13

$$
\mathcal{L}=\frac{f_{\pi}^{2}}{4} \operatorname{tr}\left(\partial_{\mu} U \partial^{\mu} U^{\dagger}\right)+\frac{1}{32 e^{2}} \operatorname{tr}\left(\left[\partial_{\mu} U, \partial_{\nu} U^{\dagger}\right]\left[\partial^{\mu} U, \partial^{\nu} U^{\dagger}\right]\right)
$$

where $e$ is the only free parameter.

The static soliton configuration of the Skyrme model is given by the celebrated hedgehog ansatz

$$
U_{0}(\boldsymbol{r})=\exp [i \boldsymbol{\tau} \cdot \hat{\boldsymbol{r}} F(r)]
$$

which introduces the chiral angle $F(r)$. Substituting this ansatz into the Lagrangian (2.1) yields minus the classical energy functional $E_{\mathrm{cl}}$. The soliton configuration is then obtained by minimizing this functional. In the Skyrme model the baryon number current is identified [13] with the topological current which classifies the mappings of the type (2.2) with the

\footnotetext{
${ }^{a}$ For a two dimensional model operator orderings were considered in ref. [15 with regard to internal symmetries.
} 
boundary condition $F(r) \stackrel{r \rightarrow \infty}{\longrightarrow} 0$. The baryon number associated with a given chiral angle, $F(r)$, is obtained as the spatial integral over the time component of this current

$$
B=\frac{2}{\pi} \int_{0}^{\infty} d r F^{\prime}(r) \sin ^{2} F(r)=-\frac{1}{\pi} F(0)
$$

Integer baryon numbers thus correspond to the boundary conditions $F(0)=n \pi$ with $n$ being a positive or negative integer. In this context it should be remarked that in topological soliton models $E_{\mathrm{cl}}$ diverges as one deviates from this boundary condition. Obviously, one crosses from the baryon to the anti-baryon sector by reversing the sign of the chiral angle, $F(r)$, since not only the baryon number but also the baryon number density acquires the opposite sign.

In order to project the soliton onto states with good spin and isopin one introduces time dependent collective coordinates parametrizing the corresponding rotations. Due to the symmetry of the hedgehog ansatz the rotations in coordinate and iso-space are equivalent. Hence one approximates the time dependent solutions by [13]

$$
U(\boldsymbol{r}, t)=A(t) U_{0}(\boldsymbol{r}) A^{\dagger}(t)
$$

The collective coordinates are contained in the $\mathrm{SU}(2)$ matrix $A(t)$. From the defining relation (1.3) we observe that the $G$-operation corresponds to the reversal of the sign of the chiral angle $F \rightarrow-F$ while keeping the collective coordinates, $A(t)$, unaltered. According to (2.3) this indicates that in the soliton sector the $G$-parity transformation actually corresponds to a particle conjugation.

A more transparent parametrization of the collective coordinates and their time dependence is given in terms of the adjoint representation matrix, $D_{a b}$, and the angular velocity, $\Omega$

$$
D_{a b}=\frac{1}{2} \operatorname{tr}\left(\tau_{a} A \tau_{b} A^{\dagger}\right) \quad \text { and } \quad A^{\dagger} \frac{\partial}{\partial t} A=\frac{i}{2} \boldsymbol{\tau} \cdot \Omega
$$

The collective Lagrangian associated with the isorotating hedgehog (2.4)

$$
L=-E_{\mathrm{cl}}+\frac{1}{2} \alpha^{2} \Omega^{2}
$$

defines the moment of inertia, $\alpha^{2}$, which again is a functional of the chiral angle, $F(r)$ m. Here we should stress that the classical mass as well as the moment of inertia do not change when the sign of the chiral angle is reversed, i.e. when one goes from the baryon to the anti-baryon. Furthermore both quantities are of the order $N_{C}$.

In the process of quantizing the collective coordinates, the spin operator is identified as the quantity canonical to the angular velocity $\Omega$

$$
\boldsymbol{J}=\frac{\partial L}{\partial \boldsymbol{\Omega}}=\alpha^{2} \boldsymbol{\Omega}
$$

For what comes later it important to note that the spin operators act as right generators in the collective space

$$
[\boldsymbol{J}, A]=\frac{1}{2} A \boldsymbol{\tau} \quad \Longrightarrow \quad\left[J_{i}, D_{a b}\right]=i \epsilon_{i b c} D_{a c} .
$$

\footnotetext{
${ }^{a}$ For explicit expressions of $E_{\mathrm{cl}}$ and $\alpha^{2}$ in various Skyrme type models the reader should consult ref. [2] and references therein.
} 
Due to the invariance of the static soliton under combined rotations in coordinate and isospace the isospin is related to the spin via the rotation

$$
I_{a}=-D_{a b} J_{b} .
$$

This restricts the possible eigenstates of the collective Hamiltonian

$$
H=E_{\mathrm{cl}}+\frac{\boldsymbol{J}^{2}}{2 \alpha^{2}}=E_{\mathrm{cl}}+\frac{J(J+1)}{2 \alpha^{2}}
$$

to those with total isospin $I=J$. Since the isospin operators correspond to the left generators one may replace

$$
D_{a b} \longrightarrow-\frac{4}{3} I_{a} J_{b}
$$

in the subspace $I=J=1 / 2$ as a consequence of the Wigner-Eckart theorem. We therefore do not need any further specification of the nucleon or anti nucleon wave-functions in the collective space.

Various static nucleon properties are obtained from the matrix elements of the vector and axial vector currents (1.1). These correspond in the Skyrme model to the symmetry currents which couple linearly to external gauge fields introduced in eqn (2.1). The currents are then given in terms of the chiral field and its derivatives. Next the rotating hedgehog configuration (2.4) is substituted and the angular velocity is eliminated with the quantization prescription (2.7). As the moment of inertia, $\alpha^{2}$ is of the order $N_{C}$ it is obvious that each such substitution reduces the order of $N_{C}$ by one unit. Finally the currents are obtained as combinations of collective operators whose coefficients are radial functions which depend on the chiral angle, $F(r)$. These combinations are displayed schematically in table 2.1. Note that due to the static nature of the soliton configuration the time $(\mu=0)$ and spatial $(\mu=i)$ components of the currents behave differently. Actually the form of currents displayed in table 2.1 is the most general one at leading order in $1 / N_{C}$ once proper account is taken the rotational symmetries in coordinate- and isospace as well as parity. Additionally one might have a time component for the axial singlet current $A_{0}^{0}=A_{0}(r) \hat{\boldsymbol{r}} \cdot \boldsymbol{J}$. However, $A_{0}(r)=0$ in Skyrme type models. We have defined the isospin part of the generator of the isoscalar currents to be $1 / N_{C}$. The proper normalization of the electromagnetic charges is guaranteed by $\int d^{3} r V_{1}(r)=1$ and $\int d^{3} r V_{3}(r)=\alpha^{2}$. The explicit forms of the radial functions $V_{1}(r), . ., A_{5}(r)$ may readily be extracted from the review articles $[1]$.

We have also listed in table 2.1, the possible sign change of the currents when one replaces $F \rightarrow-F$ in the explicit forms of the radial functions. According to our discussion above this sign change should represent the $G$-parity of the appropriate current. In every case, this is seen to agree with (1.2) in the underlying QCD theory.

It is important to note that in the transition from the classical objects $D_{a b}$ and $\Omega$ to the quantum operators $D_{a b}$ and $\boldsymbol{J}$ there can be ordering ambiguities in cases where the product of two operators is required. Such products appear for the time components of the isovector-vector current, $V_{0}^{a}$, and the isovector-axial vector current, $A_{0}^{a}$. For the former there is no ambiguity because $\left[D_{a b}, J_{b}\right]=0$. For the latter we must choose a symmetric ordering to preserve the hermitean nature of the current. Using the substitution (2.11) shows that matrix elements of $A_{0}^{a}$ vanish between nucleon states. This has the desired consequence that the $G$-parity violating form factor $G_{T}\left(\boldsymbol{q}^{2}\right)$ in the Lorentz covariant decomposition of the matrix elements

$$
\left\langle N^{\prime}\left|A_{\mu}^{a}\right| N\right\rangle=\bar{u}\left(\boldsymbol{p}^{\prime}\right)\left[G_{A}\left(\boldsymbol{q}^{2}\right) \gamma_{\mu}+\frac{G_{p}\left(\boldsymbol{q}^{2}\right)}{2 M} q_{\mu}+\frac{G_{T}\left(\boldsymbol{q}^{2}\right)}{2 M} i \sigma_{\mu \nu} q^{\nu}\right] \gamma_{5} \frac{\tau_{a}}{2} u(\boldsymbol{p}), q_{\mu}=p_{\mu}^{\prime}-p_{\mu}
$$


Table 2.1: The structure of the symmetry currents in chiral soliton models, the power of the leading term in an $1 / N_{C}$ expansion as well as the behavior of the radial functions under sign reversal of the chiral angle and their $G$-parity.

\begin{tabular}{|c|cccc|}
\hline Current & Structure & $\mathcal{O}\left(N_{C}\right)$ & $F \leftrightarrow-F$ & $G$-parity \\
\hline$V_{0}^{0}$ & $V_{1}(r)$ & 0 & - & - \\
$V_{i}^{0}$ & $V_{2}(r) \epsilon_{i j k} \hat{r}_{j} J_{k} / \alpha^{2}$ & -1 & - & - \\
$V_{0}^{a}$ & $-V_{3}(r) D_{a b} J_{b} / \alpha^{2}=V_{3}(r) I_{a} / \alpha^{2}$ & 0 & + & + \\
$V_{i}^{a}$ & $V_{4}(r) \epsilon_{i j k} \hat{r}_{j} D_{a k}$ & 1 & + & + \\
\hline$A_{0}^{0}$ & 0 & & & + \\
$A_{i}^{0}$ & $\left(A_{1}(r) \delta_{i j}+A_{2}(r) \hat{r}_{i} \hat{r}_{j}\right) J_{j} / \alpha^{2}$ & -1 & + & + \\
$A_{0}^{a}$ & $A_{3}(r) \epsilon_{i j k} \hat{r}_{i}\left\{D_{a j}, J_{k}\right\} / \alpha^{2}$ & 0 & - & - \\
$A_{i}^{a}$ & $\left(A_{4}(r) \delta_{i j}+A_{5}(r) \hat{r}_{i} \hat{r}_{j}\right) D_{a j}$ & 1 & - & - \\
\hline
\end{tabular}

is zero in the Skyrme model ( $c f$. appendix C of ref. 18]). If we had incorrectly chosen a different ordering for the operators, $A_{0}^{a}$ would not have vanished between nucleon states and a non zero $G_{T}\left(\boldsymbol{q}^{2}\right)$ form factor would have resulted, violating $G$-parity. This provides an illustration of the connection between proper operator ordering and $G$-parity invariance. At this stage we see that a symmetric, or hermitean, ordering of the collective operator is in accordance with the $G$-parity symmetry.

Returning to the discussion of table 2.1 we again stress that when going from the baryon to the anti-baryon sector, i.e. $F \leftrightarrow-F$, the symmetry currents acquire a sign according to their $G$-parity quantum numbers. This just reflects the fact that the number of pions (as measured by the power in which $F$ appears) is either even or odd for a given current. Of course, all extensions of the model should maintain this property. Furthermore, operator ordering ambiguities of a current, which may occur at higher order in the $1 / N_{C}$ expansion, have to be resolved in such a way that the phase acquired under $F \leftrightarrow-F$ is determined by the $G$-parity quantum number of the current.

This prescription has a fruitful application for the axial-singlet current, which is especially interesting in the context of the proton spin puzzle. Not long ago it was proposed that an axial-singlet current of the form

$$
A_{\mu}^{0}=i c \operatorname{tr}\left(U \partial_{\nu} U^{\dagger} U \partial_{\mu} U^{\dagger} U \partial^{\nu} U^{\dagger}\right)=i c \operatorname{tr}\left(p_{\nu} p_{\mu} p^{\nu}\right)
$$

(resulting from an additional term in (2.1)) would lead to a non-vanishing matrix element between proton states [19]. The anti-Hermitian pseudovector $p_{\mu}$ is given by $p_{\mu}=\partial_{\mu} \xi \xi^{\dagger}+\xi^{\dagger} \partial_{\mu} \xi$ with $\xi$ denoting a root of the chiral field, i.e. $U=\xi^{2}$. The reversal $F \leftrightarrow-F$ corresponds to $\xi \leftrightarrow \xi^{\dagger}$ and thus $p_{\mu} \leftrightarrow-p_{\mu}$. It is then obvious [21] that, unless the current (2.13) vanishes identically, it contradicts the $G$-parity symmetry, $c f$. table 2.1. Let us see, however, how an improper ordering can lead to a non-vanishing result. As $p_{\mu}=p_{\mu}^{a} \tau_{a}$ is a vector in iso-space, (2.13) can be expressed as $2 i \epsilon_{a b c} p_{\nu}^{a} p_{\mu}^{b} p^{\nu c}$ which vanishes as long as the $p_{\mu}^{a}$ are considered to be classical objects. Under the canonical quantization (2.7) the angular velocity, $\Omega$, which is contained in $p_{0}$, is replaced by $\boldsymbol{J} / \alpha^{2}$. Then one is tempted to replace $\epsilon_{a b c} \Omega_{b} \Omega_{c}$ by $\epsilon_{a b c} J_{b} J_{c} /\left(\alpha^{2}\right)^{2}=i J_{a} /\left(\alpha^{2}\right)^{2}$. This in turn would yield a non-vanishing axial-singlet current

$$
A_{i}^{0}=\frac{2 c}{\left(\alpha^{2}\right)^{2}} F^{\prime}(r) \sin ^{2} F(r) \hat{\boldsymbol{r}} \cdot \boldsymbol{J} \hat{r}_{i}=\frac{4 \pi^{2} c}{\left(\alpha^{2}\right)^{2}} r^{2} V_{1}(r) \hat{\boldsymbol{r}} \cdot \boldsymbol{J} \hat{r}_{i}
$$


However, (2.14) is suspicious on a priori grounds since it changes sign on $F \rightarrow-F$, in disagreement with the requirement that $A_{i}^{0}$ have positive $G$-parity. We note that the hermitean ordering

$$
\Omega_{a} \Omega_{b} \longrightarrow \frac{1}{2}\left\{\frac{J_{a}}{\alpha^{2}} \frac{J_{b}}{\alpha^{2}}+\frac{J_{b}}{\alpha^{2}} \frac{J_{a}}{\alpha^{2}}\right\}
$$

causes the axial-singlet current to vanish, in agreement with its $G$-parity transformation property. Note also that the expression (2.14) is of order $1 / N_{C}^{2}$ and thus not of leading order in the $1 / N_{C}$ expansion.

Thus the studies in the simple Skyrme model indicate that an hermitean ordering resolves the ambiguities, which occur in the transition from the classical to the quantum level, in agreement with the constraints imposed by the $G$-parity symmetry.

\section{Including Vector Mesons}

It is well known that the inclusion of vector mesons improves many predictions of the simple Skyrme model. The too small value of $g_{A}$ is slightly increased but the problem remains. Therefore one is naturally curious about possible $\mathcal{O}(1)$ corrections to the leading $\mathcal{O}\left(N_{C}\right)$ expression for $A_{i}^{a}$ (see table 2.1). In this section, the $G$-parity of the currents in the model with vector mesons is studied. It is shown that if an arbitrary operator ordering were to be allowed then $\mathcal{O}(1)$ corrections to both $g_{A}$ and the isovector magnetic moment, $\mu_{V}$ would appear to exist. However, such contributions are seen to be $G$-parity violating and hence must be eliminated by an appropriate operator ordering.

In the presence of vector mesons the situation is more involved not only because the static energy functional, $E_{\mathrm{cl}}$ contains these additional fields but also because field components, which vanish classically, get excited by the collective rotation (2.4). At this point we will not present the detailed structure of the Lagrangian we are considering. Its form has already been proposed a decade ago [20]. Also the static soliton configuration has been constructed [21] and the fields induced by the collective rotation have been computed [22]. These allowed for a reasonable description of static baryon properties even in the three flavor model [23]. Here we rather wish to discuss the $G$-parity properties of the currents. Besides the pseudovector $p_{\mu}$, which is defined after eqn (2.13), we need the isovector-vector $v_{\mu}=\xi^{\dagger} \partial_{\mu} \xi-\partial_{\mu} \xi \xi^{\dagger}$. Obviously $v_{\mu}$ is even under $G$-parity. The vector meson fields are most conveniently parametrized in terms of

$$
R_{\mu}=\omega_{\mu}+\rho_{\mu}-\frac{i}{2 g} v_{\mu}
$$

The coupling constant $g$ is determined from the decay width of the process $\rho \rightarrow \pi \pi$. As the isoscalar-vector field $\omega$ is odd under $G$-parity no definite $G$-parity quantum number can be attached to $R_{\mu}$. The isovector-vector field $\rho_{\mu}$ possesses the same quantum numbers as $v_{\mu}$. In terms of these quantities (and the field tensor for the vector meson fields) the symmetry currents of the model under consideration are displayed in eqn (2.12) of ref. [23]. There are many terms so we will explicitly display here just two representative ones for the axial vector current:

$$
\begin{aligned}
A_{\mu}^{a}= & -\frac{m_{V}^{2}}{g} \operatorname{tr}\left\{\frac{\tau_{a}}{2}\left[\xi R_{\mu} \xi^{\dagger}-\xi^{\dagger} R_{\mu} \xi\right]\right\} \\
& +\frac{i \gamma_{1}}{6} \epsilon_{\mu \nu \rho \sigma} \operatorname{tr}\left\{\frac { \tau _ { a } } { 2 } \left[\xi\left(R^{\nu} p^{\rho} p^{\sigma}+p^{\nu} p^{\rho} R^{\sigma}-p^{\nu} R^{\rho} p^{\sigma}\right) \xi^{\dagger}\right.\right.
\end{aligned}
$$


Table 3.1: The behavior of the vector meson radial functions under $F \rightarrow-F$ and the $G^{-}$ parity quantum number of these fields.

$$
\begin{array}{r|cc|ccc|}
\hline & \omega(r) & G(r) & \Phi(r) & \xi_{1}(r) & \xi_{2}(r) \\
\hline F \rightarrow-F & - & + & - & + & + \\
G \text {-parity } & - & + & - & + & + \\
& \left.\left.+\xi^{\dagger}\left(R^{\nu} p^{\rho} p^{\sigma}+p^{\nu} p^{\rho} R^{\sigma}-p^{\nu} R^{\rho} p^{\sigma}\right) \xi\right]\right\}+\ldots .
\end{array}
$$

Here $m_{V}$ denotes the vector meson mass while the coupling constant $\gamma_{1}$ can be determined from anomalous decays like $\omega \rightarrow \pi \pi \pi$ [21]. Since $g_{A}$ is given by the matrix element of $\int d^{3} r 2 A_{3}^{3}$ between proton states with spin projection $+\frac{1}{2}$ we only need to consider the spatial components $A_{i}^{a}$. In order to discuss the $G$-parity properties of $A_{i}^{a}$, we need both the static as well as the excited vector meson fields. The static hedgehog ansätze read

$$
\omega_{0}(\boldsymbol{r})=\frac{1}{2 g} \omega(r) \quad \text { and } \quad \rho_{i}^{a}(\boldsymbol{r})=\frac{G(r)}{g r} \epsilon_{i k a} \hat{r}_{k}
$$

while all other field components vanish. The equations of motion for the radial functions $\omega(r)$ and $G(r)$ as obtained from minimizing the classical energy functional are, in general, non-linear inhomogeneous differential equations. The source for $\omega(r)$ is of the form $V_{1}(r)$ (cf. table 2.1) while $G(r) \sim \epsilon_{i k a} \hat{r}_{k} \operatorname{tr}\left(\tau_{a} v_{i}\right)$. Hence $\omega(r)$ changes its sign under the reversal $F \rightarrow-F$ but $G(r)$ does not. Thus we find that again the transformation $F \rightarrow-F$ gives the proper $G$-parity quantum number. Upon the collective isorotation (2.4) we have not only $\rho_{i}^{a}(\boldsymbol{r}) \rightarrow A(t) \rho_{i}^{a}(\boldsymbol{r}) A^{\dagger}(t)$, but, as already mentioned, additional fields are induced

$$
\left.\omega_{i}=\frac{\Phi(r)}{2 g} \epsilon_{i j k} \Omega_{j} \hat{r}_{k} \quad \text { and } \quad \rho_{0}=\frac{1}{2 g} A(t) \boldsymbol{\tau} \cdot\left[\xi_{1}(r) \boldsymbol{\Omega}+\xi_{2}(r)(\hat{\boldsymbol{r}} \cdot \boldsymbol{\Omega}) \hat{\boldsymbol{r}}\right]\right) A^{\dagger}(t) .
$$

It is important to remark that the ordering between $A(t)$ and $\Omega\left(\rightarrow \boldsymbol{J} / \alpha^{2}\right)$ is completely arbitrary because they are considered to be commuting $c$-number quantities. The radial functions $\xi_{1}(r), \xi_{2}(r)$ and $\Phi(r)$ solve linear inhomogeneous differential equations which serve to extremize the moment of inertia, $\alpha^{2}$. The classical fields act as inhomogeneous parts in these equations. Hence one can deduce the behavior of the induced fields under $F \rightarrow-F$. It turns out that $\xi_{1}(r)$ and $\xi_{2}(r)$ are even while $\Phi(r)$ is odd, in agreement with $G$-parity of these fields. These results are summarized in table 3.1. It is, of course, not surprising that the behavior of the vector mesons under $F \rightarrow-F$ coincides with their $G$-parity as the underlying Lagrangian possesses this symmetry and the vector mesons couple according to their isospin and spin quantum numbers to the currents displayed in table 2.1.

Now we are completely equipped to discuss possible ordering ambiguities for $g_{A}$ and their eventual resolution by demanding the proper $G$-parity behavior. We will see that up to nextto leading order in the $1 / N_{C}$ expansion no contradiction to the $G$-parity symmetry appears as long as we consider all fields to be classical objects. Due to its isoscalar character the $\omega$ meson does not contribute in the first term in eqn (3.2), which is "non-anomalous" (i.e. no $\epsilon$-symbol). It is thus obvious that this term is odd under $G$-parity just as the isovector-axial vector current is supposed to be. For the anomalous term the situation is somewhat more involved. One can convince oneself that for this term the proper $G$-parity is obtained only when $R$ is odd, $i$. $e$. only the $\omega$ meson is allowed. Since we are taking $\mu=i$, one of the 
three remaining indices has to be associated with the time coordinate. Let us first assume that this is attached to the $\omega$ field, which is classical. The $p$ 's are classical as well because only their spatial components contribute. Thus the total contribution from the anomalous terms to $A_{i}^{a}$ is classical and no ordering ambiguities appear when the time like coordinate is assigned to the $\omega$ meson. This, of course, is nothing but the leading order part of $A_{i}^{a}$ in the $1 / N_{C}$ expansion. It contributes to the radial functions $A_{4}(r)$ and $A_{5}(r)$ in table 2.1 and has been studied in detail [22, 23]. What happens when the $\omega$ field is spatial? In that case the contribution of the anomalous term to $A_{i}^{a}$ contains two powers of $\boldsymbol{\Omega}$ since one of the two $p$ 's contains a time derivative. Hence this term is of next-to-next-to leading order in the $1 / N_{C}$ expansion and is commonly neglected.

A next-to leading order term can be obtained only when the isovector part of $R^{\nu}$ is considered and the angular velocity is treated as an operator in the collective space. Let us see how this comes about. A straightforward calculation assuming the apparently natural ordering indicated in (3.4) leads to

$$
g_{A}^{(1)}=\frac{16 \pi}{9 g} \gamma_{1} \int d r\left[r F^{\prime}\left(G+\xi_{1}\right) \sin F-\left(\xi_{1}+\xi_{2}\right) \sin ^{2} F\right]\left\langle\operatorname{tr}\left(\frac{\tau_{3}}{2} A \Omega_{3} A^{\dagger}\right)\right\rangle_{N}
$$

where the matrix element with respect to the nucleon state is indicated. Classically the trace in (3.5) vanishes identically. However, if one replaced $\Omega_{3}$ by $J_{3} / \alpha^{2}$ this would no longer by the case. Instead one would find by using (2.8) and (2.11)

$$
\left\langle\operatorname{tr}\left(\frac{\tau_{3}}{2} A \Omega_{3} A^{\dagger}\right)\right\rangle_{N} \rightarrow \frac{1}{2 \alpha^{2}}\left\langle\operatorname{tr}\left(\frac{\tau_{3}}{2}\left[A, J_{3}\right] A^{\dagger}\right)\right\rangle_{N}=\frac{-1}{4 \alpha^{2}}\left\langle D_{33}\right\rangle_{N}=\frac{-1}{12 \alpha^{2}} .
$$

However, this result cannot be taken seriously since the integrand in eqn (3.5) has the incorrect transformation property when going from the nucleon to the anti-nucleon. According to table 2.1 the integrand should be odd under $F \rightarrow-F$ but it is even as can be observed from table 3.1. Physically $g_{A}^{(1)} \neq 0$ implies that the decay amplitude for the $\beta$-decay of the anti-neutron is different in magnitude from that of the neutron. This, of course, contradicts our present understanding of physics. Hence we conclude that the collective operators have to be ordered such that (3.5) vanishes identically.

Here we have just considered two terms of the complete axial vector current in the vector meson model. The above arguments, however, apply as well to all terms in (2.12) of ref. [23]. Furthermore one may conduct the same studies for the isovector-vector current in this model. The spatial components of this current give the isovector part of the magnetic moment $\mu_{V}$. As expected, the leading order in the $1 / N_{C}$ expansion is free of ambiguities and agrees with the $G$-parity constraints. At the next-to leading order ambiguities for the collective operators occur. Again the improper behavior of the radial part of the matrix element under $F \rightarrow-F$ forces one to arrange these operators so that the $1 / N_{C}$ correction to $\mu_{V}$ vanishes.

The lesson we learn from studying the symmetry currents in the vector meson model is that matrix elements which are different from zero only because of the commutation relations (2.8) but vanish classically are likely to violate the $G$-parity symmetry. In that event those matrix elements should be discarded.

\section{Chiral Quark Solitons}

The results found in the vector meson model make us suspicious about the recently discovered $1 / N_{C}$ corrections to $g_{A}$ and $\mu_{V}$ [4]- [9] since they depend on operator ordering. Especially, it has been noticed that both the vector meson addition and the quark addition 
to the non-linear $\sigma$ model similarly describe short distance corrections to the pion cloud. This is true both for the problems of the neutron-proton mass difference [24] and the matrix element of the axial singlet current [25].

Unfortunately the effect of the reversal $F \rightarrow-F$ is not as clear in quark soliton models as it is in purely mesonic models. We therefore divide the present section into two parts. In the first one we set up the chiral quark model for both the nucleon and the anti-nucleon sectors. We derive a prescription to transform the quark spinors between these two sectors (cf. (4.9)). In the second part this is applied to the investigation of the behavior of the $1 / N_{C}$ corrections to $g_{A}$ and $\mu_{V}$ under $F \rightarrow-F$. Technical details of these studies are presented in appendix A.

The chiral quark model [26, 27], the simplest chirally symmetric model to contain quark solitons, is defined as the sum of the non-linear $\sigma$-model Lagrangian with a pion mass term

$$
\mathcal{L}_{\mathrm{nl} \sigma}=\frac{f_{\pi}^{2}}{4} \operatorname{tr}\left(\partial_{\mu} U \partial^{\mu} U^{\dagger}\right)+\frac{m_{\pi}^{2} f_{\pi}^{2}}{4} \operatorname{tr}\left(U+U^{\dagger}-2\right)
$$

and a valence quark field $\Psi_{\text {val }}$ in the background of the chiral field $U$

$$
\mathcal{L}_{\mathrm{q}}=\bar{\Psi}_{\text {val }}\left(i \not \partial-m U^{\gamma_{5}}\right) \Psi_{\text {val }}
$$

Here the mass $m=g_{\mathrm{q}} f_{\pi}$ represents a convenient parametrization of the coupling $g_{\mathrm{q}}$ between the valence quark and the chiral field. In this model the eventual effects of sea quarks are assumed to be represented by the kinetic term in (4.1). It is also obvious that the model is formulated locally.

Again we employ the hedgehog ansatz (2.2) for the pseudoscalar fields. For this static configuration the Dirac equation becomes an eigenvalue problem

$$
h(F) \Psi_{\mathrm{val}}=\epsilon_{\mathrm{val}} \Psi_{\mathrm{val}}
$$

which defines the Dirac Hamiltonian

$$
h(F)=\boldsymbol{\alpha} \cdot \boldsymbol{p}+\beta m\left[\cos F(r)+i \gamma_{5} \boldsymbol{\tau} \cdot \hat{\boldsymbol{r}} \sin F(r)\right]
$$

as a function of the chiral angle, $F(r)$. This Dirac Hamiltonian commutes with the grand spin

$$
\boldsymbol{G}=\boldsymbol{l}+\frac{\boldsymbol{\tau}}{2}+\frac{\boldsymbol{\sigma}}{2}
$$

and the parity $(\Pi)$ operators. Thus the eigenstates are classified by $G^{\Pi_{\text {int }}}$ where $\Pi_{\text {int }}$ refers to the intrinsic parity, which is defined via $\Pi=\Pi_{\text {int }}(-1)^{G}$. The construction of these eigenstates is described in appendix A.

The eigenvalue $\epsilon_{\text {val }}$ turns out to be a functional of $F$, as does the total classical energy

$$
E_{\mathrm{cl}}=E_{\mathrm{nl} \sigma}+\operatorname{sgn}(B) N_{C} \epsilon_{\mathrm{val}}
$$

with $E_{\mathrm{nl} \sigma}=2 \pi f_{\pi}^{2} \int d r\left(r^{2} F^{2}+2 \sin ^{2} F+2 m_{\pi}^{2}(1-\cos F)\right)$. The sign of the baryon number has been included in order to accommodate the hole interpretation of the Dirac theory. In a moment we will see that this relative sign is also obtained by requiring that the nucleon and anti-nucleon possess equal masses. 
The soliton is computed by solving the Euler-Lagrange equation for the chiral angle

$$
F^{\prime \prime}=-\frac{2}{r} F^{\prime}+\frac{\sin 2 F}{r^{2}}+m_{\pi}^{2} \sin F-\operatorname{sgn}(B) \frac{N_{C} m}{f_{\pi}^{2}} \int \frac{d \Omega}{4 \pi} \Psi_{\text {val }}^{\dagger} \beta\left(\sin F-i \gamma_{5} \hat{\boldsymbol{r}} \cdot \boldsymbol{\tau} \cos F\right) \Psi_{\text {val }}
$$

self-consistently. For the boundary condition $F(0)=-\pi$ the bound valence quark state of the self-consistent solution is found in the $G^{\pi_{\text {int }}}=0^{+}$channel. We denote the corresponding eigenvalue and eigenfunction by $\epsilon_{\text {val }}^{+}$and $\Psi_{\text {val }}^{+}$, respectively. On the other hand, when assuming the boundary condition $F(0)=\pi$, a strongly bound quark is only obtained in the $0^{-}$channel with eigenvalue $\epsilon_{\mathrm{val}}^{-}$and eigenfunction $\Psi_{\mathrm{val}}^{-}$. This, of course, reflects nothing but the fact that the parities of the nucleon and the anti-nucleon are opposite. In fact the self-consistent solutions for the nucleon $(F(0)=-\pi)$ and anti-nucleon $(F(0)=\pi)$ are distinguished by different overall signs of $F(r)$ and the eigenvalues of the bound quarks, i.e. $\epsilon_{\mathrm{val}}^{-}=-\epsilon_{\mathrm{val}}^{+}$. Thus, due to the inclusion of the factor $\operatorname{sgn}(B)$ in (4.6), the classical masses of the baryon and anti-baryon are identical.

Actually, the self-consistent solution for the anti-baryon can easily be obtained from the one for the baryon by noting that

$$
h(-F)=-\mathcal{J}^{\dagger} h(F) \mathcal{J} \quad \text { with } \quad \mathcal{J}=\mathcal{J}^{\dagger}=i \beta \gamma_{5}
$$

The transformation $\mathcal{J}$ commutes with $\boldsymbol{G}$ but has negative parity. This implies for the eigenvalues and eigenstates of the Dirac Hamiltonian

$$
\epsilon_{\mu}^{G^{ \pm}} \stackrel{F \rightarrow-F}{\longrightarrow}-\epsilon_{\mu}^{G^{\mp}} \quad \text { and } \quad\left|\mu, G^{ \pm}\right\rangle \quad \stackrel{F \rightarrow-F}{\longrightarrow} \quad\left|\mu, G^{\mp}\right\rangle=\mathcal{J}\left|\mu, G^{ \pm}\right\rangle,
$$

where $\mu$ labels the particular eigenstate. Details of the transformation (4.9) in terms of the radial parts of the quark wave-functions are given in appendix A. We will make extensive use of this transformation when comparing the currents for the nucleon and anti-nucleon. However, we first have to perform the projection of the chiral quark soliton onto states with good spin and isospin. For the non-linear $\sigma$ model this is straightforwardly achieved by substituting the rotating hedgehog (2.4) into the defining equation (4.1). This yields the mesonic part of the moment of inertia

$$
\alpha_{\mathrm{m}}^{2}=\frac{8 \pi}{3} f_{\pi}^{2} \int d r r^{2} \sin ^{2} F
$$

which obviously is identical for the baryon and the anti-baryon. In order to compute the quarks' contribution to $\alpha^{2}$ we employ the cranking method [28]. Then the quark spinors are rotating in isospace, which adds the Coriolis term $(1 / 2) \boldsymbol{\tau} \cdot \boldsymbol{\Omega}$ as a perturbation to the Dirac Hamiltonian (4.4). The energy eigenvalue acquires a change in second order perturbation, which (due to isospin invariance) may be written as $\epsilon_{\mathrm{val}} \rightarrow \epsilon_{\mathrm{val}}+(1 / 2) \alpha_{\mathrm{q}}^{2} \Omega^{2}$. Here

$$
\alpha_{\mathrm{q}}^{2}=\alpha_{\mathrm{q}}^{2}[F]=\frac{N_{C}}{2} \sum_{\mu \neq \mathrm{val}} \frac{\left|\left\langle\mu\left|\tau_{3}\right| \mathrm{val}\right\rangle\right|^{2}}{\epsilon_{\mathrm{val}}^{+}-\epsilon_{\mu}}
$$

denotes the quarks' contribution to the moment of inertia of the nucleon. As $\left[\mathcal{J}, \tau_{i}\right]=0$ one finds that $\alpha_{\mathrm{q}}^{2}[F]$ changes sign under the transformation (4.9), $\alpha_{\mathrm{q}}^{2}[-F]=-\alpha_{\mathrm{q}}^{2}[F]$. Taking into account that (4.11) represents a perturbation to valence quark energy, which enters the total energy with the overall factor $\operatorname{sgn}(B)$, the total moment of inertia

$$
\alpha^{2}=\alpha_{\mathrm{m}}^{2}+\operatorname{sgn}(B) \alpha_{\mathrm{q}}^{2}
$$


is seen to be invariant under $F \rightarrow-F$. This guarantees equal masses for the baryons and anti-baryons in the presence of rotational corrections.

In the context of the cranking method the induced components of the valence quarks are computed in a perturbation expansion in powers of $\boldsymbol{\Omega}$. This will serve to make the discussion of the particle conjugation properties of their contribution to the currents more transparent than in a variational approach [29]. In order to be consistent with the expansion for the energy we require the first order expression for the cranked wave-function

$$
\Psi_{\text {crank }}=A(t)\left\{\Psi_{\mathrm{val}}+\frac{1}{2} \sum_{\mu \neq \text { val }} \Psi_{\mu} \frac{\langle\mu|\boldsymbol{\tau} \cdot \Omega| \mathrm{val}\rangle}{\epsilon_{\mathrm{val}}-\epsilon_{\mu}}\right\} .
$$

The isospin matrices $\boldsymbol{\tau}$ carry unit grand spin and are positive under parity. Thus $|\mu\rangle \in 1^{ \pm}$ for $F(0)= \pm \pi$.

We now turn to the discussion of the currents in the chiral quark model. These are again sums of mesonic and quark parts. As they are the Noether currents of the underlying theory the overall factor $\operatorname{sgn}(B)$ is carried along for the quark part

$$
V_{\mu}^{a}\left(A_{\mu}^{a}\right)=\frac{i}{2} f_{\pi}^{2} \operatorname{tr}\left\{\frac{\tau_{a}}{2}\left(\xi p_{\mu} \xi^{\dagger} \mp \xi^{\dagger} p_{\mu} \xi\right)\right\}+\operatorname{sgn}(B) \bar{\Psi}_{\text {crank }} \gamma_{\mu}\left(\gamma_{5}\right) \frac{\tau^{a}}{2} \Psi_{\text {crank }} .
$$

The interesting part is, of course, the one which is due to the quarks, especially the spatial components $\left(\mathcal{Q}_{i}^{a}=\alpha_{i}\left(\sigma_{i}\right) \tau_{a} / 2\right)$

$$
\begin{aligned}
& N_{C} \Psi_{\text {crank }}^{\dagger} \mathcal{Q}_{i}^{b} \Psi_{\text {crank }}=N_{C} D_{a b} \Psi_{\text {val }}^{\dagger} \mathcal{Q}_{i}^{b} \Psi_{\text {val }} \\
& \quad+\frac{N_{C}}{2}\left(D_{a b} \Omega_{j}\right) \sum_{\mu \neq \text { val }} \frac{\left\langle\mu\left|\tau_{j}\right| \text { val }\right\rangle}{\epsilon_{\text {val }}-\epsilon_{\mu}} \Psi_{\text {val }}^{\dagger} \mathcal{Q}_{i}^{b} \Psi_{\mu}+\frac{N_{C}}{2}\left(\Omega_{j} D_{a b}\right) \sum_{\mu \neq \text { val }} \frac{\left\langle\text { val }\left|\tau_{j}\right| \mu\right\rangle}{\epsilon_{\text {val }}-\epsilon_{\mu}} \Psi_{\mu}^{\dagger} \mathcal{Q}_{i}^{b} \Psi_{\text {val }}
\end{aligned}
$$

In this expression we have temporarily adopted the ordering between $D_{a b}$ and $\Omega$ suggested by the form of the perturbed wave-function (4.13). In the framework of the chiral quark model the $1 / N_{C}$ corrections to $g_{A}$ and $\mu_{V}$, which were recently discussed in various similar models $[4,5,6,0,9]$, correspond to the replacement $\Omega \rightarrow \boldsymbol{J} / \alpha^{2}$ in the ordering of (4.15).

To obtain $g_{A}$ we calculate the integral $\int d^{3} r A_{3}^{3}$. The contribution from the next to leading order involves the sum over the grand spin projection (in which the energy eigenvalues are degenerate)

$$
\sum_{M}\left\langle\mathrm{val}, \pm\left|\sigma_{3} \tau_{b}\right| \mu, 1 M, \mp\right\rangle\left\langle\mu, 1 M, \mp\left|\tau_{j}\right| \mathrm{val}, \pm\right\rangle=\frac{i}{3} S_{\mu}^{ \pm} L_{\mu}^{ \pm} \epsilon_{3 b j}
$$

The signs appearing in the state vectors label the intrinsic parity. The upper (lower) sign refers to the (anti) nucleon sector. We refer to appendix A for the actual computation of these matrix elements and the definition of the integrals on the $R H S$. Since these matrix elements are proportional to the anti-symmetric $\epsilon$-tensor the appearance of the commutator $\left[D_{a b}, J_{j}\right]$ is obvious (see (2.8)) when replacing the angular velocity by the spin operator. Taking account of this prescription one finds for the axial charge of either the nucleon or the anti-nucleon

$$
\begin{aligned}
g_{A}=g_{A}^{(0)}+g_{A}^{(1)}= & \frac{8 \pi}{9} f_{\pi}^{2} \int d r r^{2}\left(F^{\prime}+\frac{\sin F}{r}\right) \\
& -\operatorname{sgn}(B) \frac{N_{C}}{3}\left(\left\langle\operatorname{val}\left|\sigma_{3} \tau_{3}\right| \operatorname{val}\right\rangle+\frac{i}{\alpha^{2}} \sum_{\mu \neq \text { val }} \frac{\left\langle\operatorname{val}\left|\tau_{1}\right| \mu\right\rangle\left\langle\mu\left|\sigma_{3} \tau_{2}\right| \text { val }\right\rangle}{\epsilon_{\text {val }}-\epsilon_{\mu}}\right) .
\end{aligned}
$$


where the $1 / N_{C}$ correction, $g_{A}^{(1)}$, is represented by the term proportional to $1 / \alpha^{2}$. We should remark that (4.17) corresponds to a spin up proton or a spin up anti-neutron which each give the same matrix element of $D_{33}$. Note that the overall sign differs in the two cases. Similar calculations give for the isovector part of the magnetic moment

$$
\begin{aligned}
\mu_{V}= & \mu_{V}^{(0)}+\mu_{V}^{(1)}=\frac{8 \pi}{9} f_{\pi}^{2} M_{N} \int d r r^{3} \sin ^{2} F \\
& -\operatorname{sgn}(B) \frac{i N_{C}}{12} M_{N}\left(\left\langle\operatorname{val}\left|\left[\boldsymbol{r} \cdot \boldsymbol{\alpha}, \sigma_{3}\right] \tau_{3}\right| \mathrm{val}\right\rangle+\frac{i}{\alpha^{2}} \sum_{\mu \neq \mathrm{val}} \frac{\left\langle\operatorname{val}\left|\tau_{1}\right| \mu\right\rangle\left\langle\mu\left|\left[\boldsymbol{r} \cdot \boldsymbol{\alpha}, \sigma_{3}\right] \tau_{2}\right| \mathrm{val}\right\rangle}{\epsilon_{\mathrm{val}}-\epsilon_{\mu}}\right) .
\end{aligned}
$$

Here the $\epsilon$-tensor, which again causes the appearance of the commutator $(2.8)$ in the next-to leading term, originates from the definition of the isovector part of the magnetic moment $\mu_{V}=1 / 2\left\langle\left(\boldsymbol{r} \times \boldsymbol{V}^{3}\right\rangle\right.$.

We have computed these matrix elements in the chiral quark model numerically. It turns out the next-to leading order is only about $1 / 10$ of the leading order. Thus these terms are more strongly suppressed than in the NJL model. This is apparently due to the fact that the chiral angle in the chiral quark model has quite a large extension yielding a larger moment of inertia.

Now we can investigate these expressions with respect to their behavior under particle conjugation. This is made simple by noting that we just have to apply the transformation (4.9). Since $\mathcal{J} \alpha_{i}=-\alpha_{i} \mathcal{J}$ and $\mathcal{J} \sigma_{i}=\sigma_{i} \mathcal{J}$ it is obvious (noting the factor $\operatorname{sgn}(B)$ ) that the leading order terms transform properly in agreement with table 2.1, i.e

$$
\begin{array}{rll}
\left\langle\text { val },+\left|\sigma_{3} \tau_{3}\right| \text { val },+\right\rangle & \stackrel{F \rightarrow-F}{\longrightarrow} & \left\langle\text { val, }-\left|\sigma_{3} \tau_{3}\right| \text { val },-\right\rangle, \\
\left\langle\text { val },+\left|\left[\boldsymbol{r} \cdot \boldsymbol{\alpha}, \sigma_{3}\right] \tau_{3}\right| \text { val, }+\right\rangle & \stackrel{F \rightarrow-F}{\longrightarrow} & -\left\langle\text { val, }-\left|\left[\boldsymbol{r} \cdot \boldsymbol{\alpha}, \sigma_{3}\right] \tau_{3}\right| \text { val },-\right\rangle,
\end{array}
$$

Since the transformation $\mathcal{J}$ does not affect the isospin structure of the matrix elements it is obvious that the matrix elements involved in the terms describing the $1 / N_{C}$ corrections transform in the same way

$$
\begin{array}{rccc}
\left\langle\mu,-\left|\tau_{1}\right| \mathrm{val},+\right\rangle\left\langle\mathrm{val},+\left|\sigma_{3} \tau_{2}\right| \mu,-\right\rangle & \stackrel{F \rightarrow-F}{\longrightarrow} & \left\langle\mu,+\left|\tau_{1}\right| \mathrm{val},-\right\rangle\left\langle\mathrm{val},-\left|\sigma_{3} \tau_{2}\right| \mu,+\right\rangle & (4.21) \\
\left\langle\mu,+\left|\tau_{1}\right| \mathrm{val},-\right\rangle\left\langle\mathrm{val},+\left|\left[\boldsymbol{r} \cdot \boldsymbol{\alpha}, \sigma_{3}\right] \tau_{2}\right| \mu,-\right\rangle & \stackrel{F \rightarrow-F}{\longrightarrow} & -\left\langle\mu,+\left|\tau_{1}\right| \text { val, }-\right\rangle\left\langle\mathrm{val},-\left|\left[\boldsymbol{r} \cdot \boldsymbol{\alpha}, \sigma_{3}\right] \tau_{2}\right| \mu,+\right\rangle .
\end{array}
$$

In appendix B we present an additional argument, which is based on the completeness of the eigenstates of the Dirac Hamiltonian, for the transformation relation (4.21). Now the $G$-parity violation of the $1 / N_{C}$ corrections becomes clear: The energy denominator in these terms changes its sign when going from the nucleon to the anti-nucleon. Thus the $1 / N_{C}$ corrections transform oppositely to the leading order contribution, i.e. incorrectly. We therefore conclude that the ordering ambiguities contained in the formulation of the quark currents (4.15) have to be resolved in such a way that the $1 / N_{C}$ corrections to $g_{A}$ and $\mu_{V}$ vanish:

$$
g_{A}^{(1)}=0 \quad \text { and } \quad \mu_{V}^{(1)}=0 .
$$

This can be achieved by demanding an hermitean ordering prescription

$$
D_{a b} \Omega_{j} \longrightarrow \frac{1}{2 \alpha^{2}}\left(D_{a b} J_{j}+J_{j} D_{a b}\right) \text {. }
$$


For this ordering each of the two subleading terms in (4.14) vanishes in the nucleon subspace after performing the sum over the grand spin projection (4.16). Since

$$
\epsilon_{k b j}\left(D_{a b} J_{j}+J_{j} D_{a b}\right)=-\frac{4}{3} I_{a} \epsilon_{k b j}\left(J_{b} J_{j}+J_{j} J_{b}\right)=0
$$

the required result (4.22) is obtained.

It is seen that the pattern obtained in the vector meson model is repeated in the chiral quark model. As already noted we are considering a local formulation of the chiral quark model. Non-local effects, as e.g. the blocking of transitions to negative energy states in (4.13), associated with the inclusion of levels other than $\mid$ val $\rangle$ in the definition (4.2) may cause different expressions for $g_{A}^{(1)}$ and $\mu_{V}^{(1)}$. We will comment on related studies [5, 7] below.

To avoid confusion, we should remark that there is no practical problem in the chiral quark model with too small $g_{A}$. In fact, $g_{A}$ comes out slightly too large in this model, although the problem can be resolved [27]by complicating the model a bit. However, the structure of the chiral quark model is, for present purposes, the same as other quark soliton models.

Let us conclude this section by commenting on the $G$-parity behavior of the axial singlet current in the chiral quark model. This has been noticed some time ago to account for the proton spin puzzle fairly well [25]. When the isospin part of the generator $\mathcal{Q}_{i}^{a}$ reduces to a unit matrix, the first term on the $R H S$ of (4.15) is easily observed to vanish. Furthermore no ordering ambiguities occur in the second and third terms because the $D$-matrix is replaced by the unit matrix. The radial part of the matrix element between nucleon states of the axial singlet current

$$
\begin{aligned}
\int d^{3} r A_{3}^{0} & =\operatorname{sgn}(B) \frac{1}{2} \sum_{\mu \neq \text { val }} \frac{\langle\text { val }|\boldsymbol{\tau} \cdot \Omega| \mu\rangle}{\epsilon_{\text {val }}-\epsilon_{\mu}}\left\langle\mu\left|\sigma_{3}\right| \text { val }\right\rangle+\text { c. c. } \\
& \stackrel{(2.7)}{\longrightarrow} \operatorname{sgn}(B) \frac{J_{i}}{2 \alpha^{2}} \sum_{\mu \neq \text { val }} \frac{\left\langle\text { val }\left|\tau_{i}\right| \mu\right\rangle}{\epsilon_{\text {val }}-\epsilon_{\mu}}\left\langle\mu\left|\sigma_{3}\right| \text { val }\right\rangle+\text { h. c. },
\end{aligned}
$$

is invariant under $F \rightarrow-F$. This result indicates that the induced part of the quark current behaves properly under $G$-parity if there happens to be no ordering ambiguity and the leading term in the $1 / N_{C}$ expansion vanishes.

\section{Summary and Conclusions}

We have formulated the particle conjugation operation, and especially its convenient realization as $G$-parity, in the framework of chiral soliton models. We specifically treated the usual Skyrme model, the Skyrme model with vector mesons and, as the simplest example containing quark fields, the chiral quark model. The prescription for $G$ conjugation in the solitonic sector is simply to reverse the sign of the profile function $F(r) \rightarrow-F(r)$ while leaving the collective coordinates $A(t)$ unchanged.

It was found that requiring the correct particle conjugation properties for currents could resolve operator ordering ambiguities which greatly affect the results of a number of interesting calculations. These ambiguities occur in the transition from the classical to the quantized formulation in the space of the collective coordinates.

In the basic Skyrme model we noted that an hermitean ordering of operators in the collective space was required to guarantee the vanishing of a $G$-parity violating axial current form factor. The same ordering also guaranteed the vanishing of the $G$-parity violating matrix element of an axial singlet current of interest in connection with the "proton spin puzzle". 
In the Skyrme model with vector mesons we investigated possible corrections down by $1 / N_{C}$ to $g_{A}$ and $\mu_{V}$. These turned out to vanish to insure $G$-parity conservation. Generally speaking, our studies indicate that objects which vanish classically due to $G$-parity should also vanish in the quantized formulation.

In the chiral quark model the situation turned out to be somewhat more involved because the quark states are not eigenstates of the particle conjugation. Having set up the formulation for the anti-nucleon we were able to show that the next-to leading order $1 / N_{C}$ corrections to $g_{A}$ and $\mu_{V}$, which are non-zero for the ordering suggested by the ansatz for the cranked quark fields (4.13), behave oppositely to their leading order counterparts under $F \rightarrow-F$. From this we concluded that the operator ordering in the space of the collective coordinates should be arranged so that these corrections vanish. It should be stressed that this conclusion follows immediately after (4.15) without reference to the quark wavefunction phase convention. This is because the identities $\alpha_{i}\left(\sigma_{i}\right) \mathcal{J}=\mp \mathcal{J} \alpha_{i}\left(\sigma_{i}\right)$ and $[\mathcal{J}, \boldsymbol{\tau}]=0$ immediately show that the leading and next-to leading order piece of the quark currents in the $1 / N_{C}$ expansion transform oppositely. Since the leading order has the correct $G$-parity behavior this type of next-to leading order corrections should actually be absent. This can be accomplished by adopting the hermitean ordering prescription (4.24) when going from the classical to the quantum level.

The results we have obtained in the chiral quark model are based on an ordinary perturbation expansion which is essentially equivalent to the cranking procedure of ref. [29]. Using a somewhat different expansion scheme for the Dirac operator including the Coriolis term the authors of refs. [5, 7] obtained a somewhat different result for the $1 / N_{C}$ correction to the axial charge

$$
-\operatorname{sgn}(B) \frac{i N_{C}}{3 \alpha^{2}} \sum_{\mu \neq \text { val }} \operatorname{sgn}\left(\epsilon_{\mu}\right) \frac{\left\langle\operatorname{val}\left|\tau_{1}\right| \mu\right\rangle\left\langle\mu\left|\sigma_{3} \tau_{2}\right| \mathrm{val}\right\rangle}{\epsilon_{\mathrm{val}}-\epsilon_{\mu}}
$$

Although this result deviates from the correction in (4.17) only slightly numerically it is obvious from our discussion that (5.1) transforms properly under the particle conjugation due to the additional factor $\operatorname{sgn}\left(\epsilon_{\mu}\right)[30]$. It should be remarked that the treatment of refs. [5, 7] is not itself free of ordering ambiguities, which for example manifest themselves in the violation of the $P C A C$ relation. Furthermore the expression (5.1) cannot be obtained from the ordinary perturbation expansion or the cranking procedure of ref. [29]. It seems to us that a number of open questions remain as to the validity of (5.1). The discussion of these goes beyond the scope of this paper nevertheless our considerations on the particle conjugation symmetry favor the results of refs. [5, 77 over those of refs. [4. 6]. The latter are essentially identical to (4.17). Stated otherwise, the considerations concerning the particle conjugation symmetry rule out the results of refs. [4, 6] while no definite statement can be made on those of refs. [5, 7]. As the models [5, 7] contain non-local effects they are somewhat different from the chiral quark model considered here.

The considerations presented in this paper seem to exclude the emergence of $1 / N_{C}$ corrections to $g_{A}$ and $\mu_{V}$ in the context of the collective quantization of the static soliton configuration when performing a "classical" expansion in the angular velocity $\Omega$ in local models. This suggests that the incorporation of pion fluctuations off the soliton is important in order to obtain non-vanishing corrections. Such a treatment seems quite appealing since it has been shown to solve problems [31] related to the fact that the current algebra relations cannot be correctly described within the collective quantization of the static soliton [32].

\footnotetext{
${ }^{a}$ We are grateful to C. Christov for pointing out this difference to us.
} 
A preliminary version of this paper has been submitted elsewhere 33].

\section{Acknowledgements}

We would like to thank R. Alkofer for helpful discussions. One of us (HW) acknowledges support by a Habilitanden-scholarship of the Deutsche Forschungsgemeinschaft (DFG). This work was supported in part by the US DOE contract number DE-FG-02-85ER 40231.

\section{Appendix A: Explicit Solution to the Chiral Quark Model}

In this appendix we present the explicit form of the eigenfunctions of the Hamiltonians $h(F)$ and $h(-F)$ for the nucleon and anti-nucleon, respectively. The Hamiltonian $h(F)$ is given in (4.4). Also the expressions for the axial charge, $g_{A}$, the isovector part of the magnetic moment, $\mu_{V}$, as well the corresponding "would-be" $1 / N_{C}$ corrections $g_{A}^{(1)}$ and $\mu_{V}^{(1)}$ are made explicit.

Although only states with grand spin $G=0$ and $G=1$ are relevant in the framework of the chiral quark model, it is helpful to define general eigenstates of the grand spin operator $G(4.5)$ :

$$
|l j G M\rangle=\sum_{j_{3} s_{3}} C_{j j_{3}, \frac{1}{2} s_{3}}^{G M} \sum_{m i_{3}} C_{l m, \frac{1}{2} i_{3}}^{j j_{3}}|l m\rangle\left|\frac{1}{2} s_{3}\right\rangle_{J}\left|\frac{1}{2} i_{3}\right\rangle_{I}
$$

Here $C_{j j_{3}, j^{\prime} j_{3}^{\prime}}^{J M}$ denote the $\mathrm{SU}(2)$ Clebsch-Gordan coefficients. The subscripts $J$ and $I$ indicate spinors in coordinate- and iso-space, respectively.

\section{Treatment of the Nucleon}

The quark state which is bound in the chiral background field $F(r)$ resides in the $G=0$ channel and possesses positive parity when the boundary condition $F(r=0)=-\pi$ is chosen. In terms of the grand spin states (A.1) this valence quark state is parametrized as

$$
|\mathrm{val},+\rangle=\left(\begin{array}{c}
i g_{0}^{+}(r)\left|0 \frac{1}{2} 00\right\rangle \\
f_{0}^{+}(r)\left|1 \frac{1}{2} 00\right\rangle
\end{array}\right) \text {. }
$$

The radial functions $g_{0}(r)$ and $f_{0}(r)$ are obtained by diagonalizing the Dirac Hamiltonian (4.4) and identifying the state with the energy eigenvalue in the interval $-m \leq \epsilon_{\mathrm{val}}^{+} \leq m$ as the valence quark. Technically the diagonalization is achieved by constraining the system to a spherical box of finite radius $D$ and imposing certain boundary conditions at $r=D$. Here we demand that the upper components of the Dirac spinor (A.2) vanish at $r=D$. For more details on the numerical treatment we refer to ref. 34.

The matrix elements relevant for the classical parts of the axial charge and the iso-vector part of the magnetic moments are easily obtained to be

$$
\begin{aligned}
\left\langle\text { val },+\left|\sigma_{3} \tau_{3}\right| \text { val },+\right\rangle & =\frac{1}{3} \int d r r^{2}\left(g_{0}^{+}(r)^{2}-\frac{1}{3} f_{0}^{+}(r)^{2}\right) \\
\left\langle\text { val },+\left|\left[\boldsymbol{r} \cdot \boldsymbol{\alpha}, \sigma_{3}\right] \tau_{b}\right| \mathrm{val},+\right\rangle & =\frac{8 i}{3} \delta_{3 b} \int d r r^{3} g_{0}^{+}(r) f_{0}^{+}(r)
\end{aligned}
$$

In order to evaluate the perturbated wave-function due to cranking we need to compute the overlap matrix element $\left\langle\mu\left|\tau_{j}\right|\right.$ val, +$\rangle$. The isospin matrix $\tau_{j}$ carries unit grand spin and 
positive parity. Thus non-vanishing matrix elements exist only when $|\mu\rangle$ belongs to the $1^{-}$ channel. These states are parametrized as

$$
|\mu, 1 M,-\rangle=\left(\begin{array}{c}
i g_{1 \mu}^{-}(r)\left|2 \frac{3}{2} 1 M\right\rangle \\
f_{1 \mu}^{-}(r)\left|1 \frac{3}{2} 1 M\right\rangle
\end{array}\right)+\left(\begin{array}{c}
i g_{2 \mu}^{-}(r)\left|0 \frac{1}{2} 1 M\right\rangle \\
-f_{2 \mu}^{-}(r)\left|1 \frac{1}{2} 1 M\right\rangle
\end{array}\right)
$$

The corresponding eigenvalues of $h(F)$ are denoted by $\epsilon_{\mu}^{-}$. Furthermore $M=-1,0,1$ refers to the projection of the grand spin $G=1$. Again we refer the interested reader to ref. [34 on details on the numerical evaluation of the radial functions $g_{1 \mu}^{+}, . ., f_{2 \mu}^{+}$. The boundary conditions on these radial functions are such that the upper component of the spinor (A.4) vanishes at $r=D$ in order to avoid finite size isospin violations [35].

It is then straightforward to compute the matrix elements relevant for the axial charge and iso-vector part of the magnetic moments, which are discussed in section 4:

$$
\begin{aligned}
\left\langle\mu, 1 M,-\left|\tau_{j}\right| \operatorname{val} \mid,+\right\rangle & =\frac{1}{2} S_{\mu}^{+}\left[\left(\tau_{j}^{11}-\tau_{j}^{22}\right) \delta_{0 M}-\sqrt{2} \tau_{j}^{12} \delta_{1 M}+\sqrt{2} \tau_{j}^{21} \delta_{-1 M}\right] \\
\left\langle\operatorname{val}|,+| \sigma_{3} \tau_{b} \mid \mu, 1 M,-\right\rangle & =\frac{1}{3 \sqrt{2}} L_{\mu}^{+}\left[\tau_{b}^{12} \delta_{-1 M}+\tau_{b}^{21} \delta_{1 M}\right] \\
\left\langle\text { val }+|| \boldsymbol{r} \cdot \boldsymbol{\alpha}, \sigma_{3}\right] \tau_{b}|\mu, 1 M,-\rangle & =\frac{-i}{3 \sqrt{2}} R_{\mu}^{+}\left[\tau_{b}^{12} \delta_{-1 M}+\tau_{b}^{21} \delta_{1 M}\right] .
\end{aligned}
$$

The coefficients $S_{\mu}^{+}, L_{\mu}^{+}$and $R_{\mu}^{+}$are given as integrals over the radial functions defined in eqns (A.3) and (A.5):

$$
\begin{aligned}
S_{\mu}^{+} & =-\int d r r^{2}\left[g_{0}^{+}(r) g_{2 \mu}^{-}(r)+f_{0}^{+}(r) f_{2 \mu}^{-}(r)\right] \\
L_{\mu}^{+} & =\int d r r^{2}\left[3 g_{0}^{+}(r) g_{2 \mu}^{-}(r)-f_{0}^{+}(r)\left(f_{2 \mu}^{-}(r)+\sqrt{2} f_{1 \mu}^{-}(r)\right)\right] \\
R_{\mu}^{+} & =\int d r r^{3}\left[g_{0}^{+}(r)\left(4 f_{2 \mu}^{-}(r)+\sqrt{2} f_{1 \mu}(r)\right)+f_{0}^{+}(r)\left(4 g_{2 \mu}^{-}(r)-\sqrt{2} g_{1 \mu}(r)\right)\right] .
\end{aligned}
$$

The summation over the grand spin projection yields

$$
\begin{aligned}
\sum_{M}\left\langle\mathrm{val},+\left|\sigma_{3} \tau_{b}\right| \mu, 1 M,-\right\rangle\left\langle\mu, 1 M,-\left|\tau_{j}\right| \mathrm{val},+\right\rangle & =\frac{i}{3} S_{\mu}^{+} L_{\mu}^{+} \epsilon_{3 b j} \\
\sum_{M}\left\langle\mathrm{val},+\left|\left[\boldsymbol{r} \cdot \boldsymbol{\alpha}, \sigma_{3}\right] \tau_{b}\right| \mu, 1 M,-\right\rangle\left\langle\mu, 1 M,-\left|\tau_{j}\right| \mathrm{val},+\right\rangle & =\frac{1}{3} S_{\mu}^{+} R_{\mu}^{+} \epsilon_{3 b j}
\end{aligned}
$$

\section{Treatment of the Anti-Nucleon}

For the boundary condition $F(r=0)=\pi$ the parity of the bound valence quark gets reversed. Thus this state is member of the $0^{-}$channel:

$$
|\mathrm{val},-\rangle=\left(\begin{array}{c}
i g_{0}^{-}(r)\left|1 \frac{1}{2} 00\right\rangle \\
f_{0}^{-}(r)\left|0 \frac{1}{2} 00\right\rangle
\end{array}\right)
$$

The corresponding eigenvalue of the Dirac Hamiltonian (4.4) is labeled $\epsilon_{\mathrm{val}}^{-}$. As the transformation $\mathcal{J}$ in eqn (4.8) exchanges upper and lower components of a Dirac spinor, we now demand the lower components of (A.8) to vanish at $r=D$. This makes possible the comparison with (A.2) even for finite $D$. 
The classical parts of $g_{A}$ and $\mu_{V}$ are given by

$$
\begin{aligned}
\left\langle\text { val, }-\left|\sigma_{3} \tau_{3}\right| \text { val },-\right\rangle & =-\frac{1}{3} \int d r r^{2}\left(f_{0}^{-}(r)^{2}-\frac{1}{3} g_{0}^{-}(r)^{2}\right) \\
\left\langle\text { val, }-\left|\left[\boldsymbol{r} \cdot \boldsymbol{\alpha}, \sigma_{3}\right] \tau_{b}\right| \text { val },-\right\rangle & =\frac{8 i}{3} \delta_{3 b} \int d r r^{3} f_{0}^{-}(r) g_{0}^{-}(r)
\end{aligned}
$$

Under the cranking perturbation the valence quark state (A.8) couples to the states in the $1^{+}$channel

$$
|\mu, 1 M,+\rangle=\left(\begin{array}{c}
i g_{1 \mu}^{+}(r)\left|1 \frac{3}{2} 1 M\right\rangle \\
-f_{1 \mu}^{+}(r)\left|2 \frac{3}{2} 1 M\right\rangle
\end{array}\right)+\left(\begin{array}{c}
i g_{2 \mu}^{+}(r)\left|1 \frac{1}{2} 1 M\right\rangle \\
f_{2 \mu}^{+}(r)\left|0 \frac{1}{2} 1 M\right\rangle
\end{array}\right)
$$

Their eigenvalues are denoted by $\epsilon_{\mu}^{+}$. Again the lower components are constrained to be zero at $r=D$. The isospin structure of the matrix elements (A.5) remains unaltered when we transfer them to the anti-nucleon. However, the coefficients $S_{\mu}^{+}, L_{\mu}^{+}$and $R_{\mu}^{+}$are replaced by

$$
\begin{aligned}
S_{\mu}^{-} & =-\int d r r^{2}\left[g_{0}^{-}(r) g_{2 \mu}^{+}(r)+f_{0}^{-}(r) f_{2 \mu}^{+}(r)\right] \\
L_{\mu}^{-} & =\int d r r^{2}\left[3 f_{0}^{-}(r) f_{2 \mu}^{+}(r)-g_{0}^{-}(r)\left(g_{2 \mu}^{+}(r)-\sqrt{2} g_{1 \mu}^{+}(r)\right)\right] \\
R_{\mu}^{-} & =\int d r r^{3}\left[g_{0}^{-}(r)\left(4 f_{2 \mu}^{+}(r)+\sqrt{2} f_{1 \mu}^{+}(r)\right)+f_{0}^{-}(r)\left(4 g_{2 \mu}^{+}(r)-\sqrt{2} g_{1 \mu}^{+}(r)\right)\right] .
\end{aligned}
$$

When the summation over the grand spin projection is performed these integrals have to be substituted for their analogous expressions in eqn (A.7).

Using the explicit representations (A.2, A.4, A.8) and (A.10) the transformation (4.9) corresponds to

$$
\begin{array}{rlrl}
g_{0}^{+}(r) & \rightarrow-f_{0}^{-}(r), & f_{0}^{+}(r) & \rightarrow g_{0}^{-}(r), \\
g_{1}^{-}(r) & \rightarrow f_{1}^{+}(r), & f_{1}^{-}(r) \rightarrow-g_{1}^{+}(r), \\
g_{2}^{-}(r) & \rightarrow-f_{2}^{+}(r), & f_{2}^{-}(r) \rightarrow g_{2}^{+}(r) .
\end{array}
$$

Numerically we have verified that the self-consistent solution reverses its $\operatorname{sign}(F \rightarrow-F)$ when we alter the boundary condition from $F(0)=-\pi$ to $F(0)=\pi$. Furthermore the computations confirm the transformations $\epsilon_{\mathrm{val}}^{+} \rightarrow-\epsilon_{\mathrm{val}}^{-}$and $\epsilon_{\mu}^{-} \rightarrow-\epsilon_{\mu}^{+}$for the eigenvalues of $h(F)$. The transformation properties for the wave-functions (A.12) are regained up to an overall, irrelevant phase. This phase may, of course, be different in the grand spin zero and one channels.

\section{Appendix B: Completeness Argument}

Using the results from appendix A the transformation relations (4.19) and (4.21) are easily verified. In terms of the integrals over the wave-functions these transformations are expressed as

$$
S_{\mu}^{+} L_{\mu}^{+} \rightarrow S_{\mu}^{-} L_{\mu}^{-} \quad \text { and } \quad S_{\mu}^{+} R_{\mu}^{+} \rightarrow-S_{\mu}^{-} R_{\mu}^{-} . \quad(\text { no sum over } \mu)
$$

The validity of the relations (4.19) and (4.21) is also nicely confirmed by making use of the completeness property of the eigenstates of the Dirac Hamiltonian. From that one can deduce

$$
\left\langle\text { val, } \pm\left|\sigma_{3} \tau_{3}\right| \text { val }, \pm\right\rangle=\frac{1}{3} \sum_{\mu} S_{\mu}^{ \pm} L_{\mu}^{ \pm} \quad \text { and } \quad\left\langle\text { val, } \pm\left|\left[\boldsymbol{r} \cdot \boldsymbol{\alpha}, \sigma_{3}\right] \tau_{3}\right| \text { val }, \pm\right\rangle=\frac{i}{3} \sum_{\mu} S_{\mu}^{ \pm} R_{\mu}^{ \pm}
$$


This indicates once again that the matrix elements involved in the subleading order of the $1 / N_{C}$ expansion transform identically to those of the leading order when the sign of the chiral angle is reversed. We should mention that we have confirmed the identities (B.2) numerically for both boundary conditions $F(0)= \pm \pi$. 
References

[1] G. Holzwarth and B. Schwesinger, Rep. Prog. Phys. 49 (1986) 825;

I. Zahed and G. E. Brown, Phys. Rep. 142 (1986) 1;

Ulf-G. Meißner, Phys. Rep. 161 (1988) 213.

[2] G. Holzwarth, Ed. Baryons as Skyrme Solitons, World Scientific, 1994.

[3] S. Brodsky, J. Ellis and M. Karliner, Phys. Lett. B206 (1988) 309;

J. Ellis and M. Karliner, Phys. Lett. B313 (1993) 131;

J. Schechter, A. Subbaraman and H. Weigel, Phys. Rev. D48 (1993) 339, and references therein.

[4] M. Wakamatsu and T. Watabe, Phys. Lett. B312 (1993) 184.

[5] A. Blotz, M. Prasałowicz and K. Goeke, Phys. Lett. B317 (1993) 195.

[6] A. Hosaka and H. Toki, Phys. Lett. B322 (1993) 1.

[7] C. V. Christov, K. Goeke, P. Pobilista, V. Petrov, M. Wakamatsu and T. Watabe, Phys. Lett. B325 (1994) 467.

[8] A. Blotz, M. Prasałowicz and K. Goeke, Axial Properties of the Nucleon with $1 / N_{C}$ Corrections in the Solitonic SU(3) NJL Model, Bochum University preprint RUB-TPII41/93, March 1994.

[9] M. Wakamatsu, Phys. Lett. B331 (1994) 425.

[10] G. Karl and J. E. Paton, Phys. Rev. D30 (1984) 238;

A. Jackson, A. D. Jackson and V. Pasquier, Nucl. Phys. A432 (1985) 567.

[11] R. Dashen, E. Jenkins and A. V. Manohar, Phys. Rev. D49 (1994) 4713.

[12] R. Alkofer and H. Weigel, Phys. Lett. B319 (1993) 1.

[13] G. S. Adkins, C. R. Nappi and E. Witten, Nucl. Phys. B228 (1993) 552.

[14] E. Tomboulis, Phys. Rev. D12 (1975) 1678.

[15] N. Dorey, J. Hughes and M. Mattis, Phys. Rev. D49 (1994) 3598;

For an application to the three dimensional Skyrme model $c f$. N. Dorey, J. Hughes and M. Mattis, Phys. Rev. D50 (1994) 5816.

[16] K. Nishijima, Prog. Theor. Phys. 6 (1951) 614, 1027;

A. Pais and R. Jost, Phys. Rev. 87 (1952) 871;

L. Michel, Nuovo Cim. 10 (1953) 319;

D. Amati and B. Vitale, Nuovo Cim. 2 (1955) 719;

C. Goebel, Phys. Rev. 103 (1956) 258;

T. D. Lee and C. N. Yang, Nuovo Cim. 10 (1956) 749.

[17] T. H. R. Skyrme, Proc. Roy. Soc. 260 (1961) 127.

[18] Ulf-G. Meißner, N. Kaiser and W. Weise, Nucl. Phys. A466 (1987) 685. 
[19] Z. Ryzak, Phys. Lett. B217 (1989) 325.

[20] Ö. Kaymakcalan and J. Schechter, Phys. Rev. D30 (1984) 594.

[21] P. Jain, R. Johnson, Ulf-G. Meißner, N. W. Park and J. Schechter, Phys. Rev. D37 (1988) 3252.

[22] Ulf-G. Meißner, N. Kaiser, H. Weigel and J. Schechter, Phys. Rev. D39 (1989) 1956.

[23] N. W. Park and H. Weigel, Nucl. Phys. A541 (1992) 453.

[24] P. Jain, R. Johnson, N. W. Park, J. Schechter and H. Weigel, Phys. Rev. D40 (1989) 855.

[25] R. Johnson, N. W. Park, J. Schechter, V. Soni and H. Weigel, Phys. Rev. D42 (1990) 2998.

[26] S. Kahana, G. Ripka and V. Soni, Nucl. Phys. A415 (1984) 351;

M. C. Birse and M. K. Banerjee, Phys. Rev. D31 (1985) 118.

[27] P. Jain, R. Johnson and J. Schechter, Phys. Rev. D38 (1988) 1571.

[28] D. R. Inglis, Phys. Rev. 96 (1954) 1059; 103 (1956) 1786.

[29] T. D. Cohen and W. Broniowski, Phys. Rev. D34 (1986) 3472.

[30] C. V. Christov, K. Goeke and P. V. Pobylitsa, " $1 / N_{c}$ Rotational Corrections to $g_{A}$ in the NJL Model and Charge Conjugation", Bochum University preprint, November 1994, RUB-TPII-15/94;

M. Wakamatsu, "On the Origin of the $g_{A}$ Problem in the Skyrme Model", Osaka University preprint, November 1994.

[31] H. Walliser, Nucl. Phys. A524 (1991) 706.

[32] N. Kaiser, U. Vogl and W. Weise, Nucl. Phys. A490 (1988) 602.

[33] J. Schechter and H. Weigel, "Particle Conjugation and the $1 / N_{C}$ Corrections to $g_{A}$ ", SU-4220-588, UNITU-THEP-23/1994, October 1994.

[34] R. Alkofer and H. Weigel, Comp. Phys. Comm. 82 (1994) 30.

[35] H. Weigel, R. Alkofer and H. Reinhardt, Nucl. Phys. B397 (1992) 638. 